\title{
Pimecrolimus Cream in the Long-Term Management of Atopic Dermatitis in Adults: A Six-Month Study
}

\author{
Michael Meurera Regina Fölster-Holst ${ }^{b}$ Gottfried Wozel ${ }^{a}$ \\ Gottfried Weidinger ${ }^{c}$ Michael J ünger ${ }^{d}$ Matthias Bräutigam ${ }^{c}$ \\ for the CASM-DE-01 Study Group \\ aHautklinik, Universitätsklinikum der TU, Dresden, bUniversitätshautklinik, Kiel, \\ cNovartis Pharma GmbH, Clinical Research, Nürnberg, dKlinik und Poliklinik für Hautkrankheiten, \\ Ernst-Moritz-Arndt-Universität, Greifswald, Deutschland
}

\section{Key Words \\ Pimecrolimus · Topical corticosteroids · Elidel ${ }^{\circledR}$. SDZ ASM 981 - Atopic dermatitis · Long-term management}

\begin{abstract}
Background: Pimecrolimus cream Elidel $^{\circledR}$, SDZ ASM 981), a non-steroid inhibitor of inflammatory cytokines, is effective in the treatment of atopic dermatitis (AD). We assessed whether early treatment of AD signs/symptoms reduces the need for topical corticosteroids. Objective: To investigate the efficacy and safety of pimecrolimus cream $1 \%$ in the long-term management of adult AD. Methods: 192 adults with moderate to severe AD were randomised (1:1) for twice daily (b.i.d.) treatment of early signs or symptoms of AD with either pimecrolimus cream $1 \%$ or vehicle cream (control group) to prevent progression to flares. Treatment was given as needed for 24 weeks. In the event of flares, a moderately potent corticosteroid (prednicarbate $0.25 \%$ cream) was permitted as rescue medication in both groups. The percentage of days on which a topical corticosteroid was used to treat
\end{abstract}

disease flares was the main outcome measure. Results: Corticosteroid medication was used on $14.2 \%$ (95\% confidence interval, $\mathrm{Cl}$ : 8.3-21.1) of the days of the 24-week treatment period in the pimecrolimus group and on $37.2 \%(95 \% \mathrm{Cl}: 30.4-44.0)$ of the days in the control group ( $p<0.001$ ). In total, $44.8 \%(43 / 96)$ of patients in the pimecrolimus group did not experience a flare compared with $18.8 \%$ (18/96) of patients in the control group. The median time to first flare was 144 days in the pimecrolimus group and 26 days in the control group ( $p<0.001$ ). Pimecrolimus treatment was also associated with improvement in signs and symptoms of AD, pruritus, patients' self-assessment and quality of life. Conclusions: Pimecrolimus cream $1 \%$ b.i.d. is an effective, well-tolerated, long-term treatment for AD in adults, substantially reducing the number of flares compared to a conventional therapy and consequently reducing or eliminating the need for corticosteroid treatment.

Copyright $($ 2002 S. Karger AG, Base

\begin{tabular}{ll}
\hline KARGER & ( ) 2002 S. Karger AG, Basel \\
1018-8665/02/2053-0271\$18.50/0 \\
$\begin{array}{l}\text { Fax + 4161306 12 34 } \\
\text { www.karger.com }\end{array}$ & $\begin{array}{l}\text { Accessible online at: } \\
\text { www.karger.com/journals/drm }\end{array}$
\end{tabular}

Prof. Dr. M. Meurer

Department of Dermatology, University Hospital

Technical University Dresden, Fetscherstrasse 74

D-01307 Dresden (Germany)

Tel. +49 351458 2497, Fax +49 351458 4338, E-Mail meurer@rcs.urz.tu-dresden.de 


\section{Introduction}

Since the 1950s, treatment of atopic dermatitis (AD) has combined the use of emollients for dry skin with reactive use of topical corticosteroids for disease flares [1, 2]. Topical corticosteroids are effective in the treatment of the acute signs and symptoms of $\mathrm{AD}$, but many patients and doctors are hesitant to use them in the long term [3, 4]. The primary concern with the long-term use of corticosteroids is the risk of local adverse effects with chronic and inappropriate use, with skin atrophy as the foremost concern $[5,6]$.

Pimecrolimus (Elidel ${ }^{\circledR}$, SDZ ASM 981) offers a novel, non-steroid approach to the future management of $\mathrm{AD}$. It is an anti-inflammatory macrolactam developed specifically for the treatment of inflammatory skin diseases [7]. Its mode of action is the selective inhibition of T-cell activation via the calcineurin pathway and inhibition of the release of inflammatory cytokines from mast cells, thereby preventing the cascade of immune and inflammatory signals that drive the development of acute and chronic $\mathrm{AD}[8,9]$. In contrast to corticosteroids, pimecrolimus has no atrophogenic potential or other steroid-specific side-effects [10].

This study investigated whether pimecrolimus cream $1 \%$, when applied at the first signs or symptoms of $\mathrm{AD}$ (e.g. mild erythema/pruritus), could prevent the disease progressing to the point at which treatment with a moderately potent topical corticosteroid would be required. With this approach, topical corticosteroids could be reserved for the treatment of acute severe disease, and the corticosteroid side-effects associated with chronic treatment could be avoided.

This was the first randomised, double-blind study designed to assess the reduction in days on topical corticosteroids in $\mathrm{AD}$ as the primary outcome measure.

\section{Participants}

\section{Study Design}

This was a randomised, double-blind, parallel-group, multicentre study in 12 dermatological university hospitals, 1 dermatological clinic and 3 dermatology practices in Germany. Patients were recruited from September 1999 to June 2000, and in total, 192 adult patients, with a clinical diagnosis of $\mathrm{AD}$ according to the criteria of Rajka [11], were randomised, 1:1, to receive treatment as needed over 24 weeks with either pimecrolimus cream or a corresponding vehicle cream, and emollients and reactive use of topical corticosteroids. Treatment was assigned by computer-generated randomisation lists. To maintain the study blind, a vehicle cream, of identical appearance and odour to the pimecrolimus cream, was used in the control group. The blinding was strictly maintained for all site monitoring and data management personnel at all times.

\section{Inclusion Criteria}

Patients were required to have $\mathrm{AD}$ affecting at least $5 \%$ of the total body surface area and an Investigator's Global Assessment (IGA) score of 3 or 4. (For definition of IGA, see primary and secondary outcome measures.)

\section{Exclusion Criteria}

Therapies which led to exclusion were: PUVA, high-dose UVA or systemic therapy with corticosteroids, immunosuppressants or cytostatics (previous 3 months); topical therapies for $\mathrm{AD}$ (previous 2 weeks); systemic antibiotics (previous 2 weeks); systemic steroids for indications other than $\mathrm{AD}$ (previous 1 month). Other exclusion criteria comprised: pregnancy or lactation; women of child-bearing age not using reliable contraception; need for treatment with potent topical steroids for control of $\mathrm{AD}$; severe concurrent allergic diseases; diseases associated with immunosuppression or malignancy; presence of skin conditions that could affect the evaluation of study treatment; active skin infections requiring treatment with a prohibited medication, or active herpes simplex infections.

\section{Planned Interventions}

Patients applied the study medication (i.e. pimecrolimus cream $1 \%$ or corresponding vehicle) to the affected areas twice daily to treat the first signs or symptoms of $\mathrm{AD}$ (e.g. pruritus, erythema) in order to prevent progression to disease flare. A flare was defined as disease status needing application of topical corticosteroid therapy for at least 3 days. The study medication was applied twice daily to the affected areas until complete clearance of signs and symptoms. Bland emollients were applied to dry skin after application of the study medication. A moderately potent topical corticosteroid, prednicarbate $0.25 \%$ cream (Dermatop ${ }^{\circledR}$ ), was administered in both groups if the patient experienced unacceptable itching and clinical signs (oozing/crusting or excessive scratch marks or severe erythema) despite treatment with the study medication. Topical corticosteroid was to be used for a maximum of 7 days b.i.d. followed by a further 7 days o.d. or until marked reduction of the signs of AD was achieved. After each course of corticosteroid there was a mandatory treatment for 7 days with the study drug (pimecrolimus cream or corresponding vehicle) to treat residual disease and prevent rebound of the disease. With the exception of cetirizine, no additional active treatment of $\mathrm{AD}$ was allowed during the study. Patients were assessed at a screening visit ( 2 weeks to 2 days prior to randomisation), at baseline and at weeks $1,3,6,12$ and 24 . There was additional telephone contact during weeks 9 and 18 and unscheduled visits in the event of flares.

\section{Ethics}

The study was performed according to Good Clinical Practice for trials on medicinal products in the European Union and the Declaration of Helsinki on medical research in humans. All patients gave written informed consent, and the Institutional Review Board of the principal investigator and the ethics committees of the individual centres approved the study protocol.

\section{Primary and Secondary Outcome Measures}

The primary efficacy endpoint was the percentage of days on which topical corticosteroid treatment was received. This endpoint reflected the design of the study in which the principal aim was to 
assess the efficacy and safety of pimecrolimus in preventing exacerbation of the disease to the extent that treatment with corticosteroid becomes necessary (i.e. a disease flare). Secondary outcome measures included the number of disease flares, the time to first flare, IGA scores, the Eczema Area and Severity Index (EASI) [12], a pruritus severity assessment and patients' self-assessment. Prior to the study, all investigators were required to undergo training in IGA and EASI assessments.

The IGA is a static (i.e. no reference to baseline state) 6-point measure of disease severity, based on an overall assessment of skin lesions: 0 (clear) $=$ no inflammatory signs of AD; 1 (almost clear $)=$ just perceptible erythema and just perceptible papulation/infiltration; $2($ mild $)=$ mild erythema and mild papulation and infiltration; $3($ moderate $)=$ moderate erythema and moderate papulation/infiltration; 4 (severe) $=$ severe disease with severe erythema and severe papulation/infiltration; 5 (very severe) $=$ severe erythema and severe papulation/infiltration with oozing/crusting.

All patients with an IGA of $\leq 2$ were classified as treatment success. The EASI is a composite, validated score objectively assessing both the severity of the four key signs of AD (erythema, infiltration/ papulation, excoriations, lichenification) in the four body regions (head/neck, trunk, upper and lower limbs) and the surface area involvement. Combining the results from each region, age-adjusted for the percentage of each body area involved, gives a total score in the range of $0-72$ [12].

In addition, the impact of treatment on patients' quality of life was assessed using two criteria, the Dermatology Life Quality Index (DLQI) and the Quality of Life Index - Atopic Dermatitis (QoLIAD). The DLQI comprises 10 questions regarding symptoms and perception of disease, daily activities, work or school, personal relationships, leisure and consequences of treatment. Each answer was scored $0-3$, giving a total index ranging from 0 (best) to 30 (worst) for quality of life. The DLQI is expressed as the percentage of the maximum possible score of 30 [13]. The QoLIAD is a questionnaire with 25 items which could be answered with yes (score $=1$ ) or no $($ score $=0$ ). The theoretical scale range is from 0 to 25 . The QoLIAD is expressed as the percentage of the maximum possible score of 25. As with the DLQI, the higher the QoLIAD score, the greater the impairment of quality of life [14].

All adverse events occurring during the study were recorded. Physical examination and standard haematology, blood chemistry and urinalysis tests were performed at regular scheduled visits throughout the study. Patients also completed diaries on medication use, changes in medical condition and pruritus (score of $0-4$ ).

\section{Statistical Analyses}

The sample size was calculated as having to include at least 172 patients ( 86 in each group). This was based on the steroid consumption $\left(\mathrm{g} / \mathrm{m}^{2}\right.$ body surface/week) seen in patients with $\mathrm{AD}$, whereby the consumption at the beginning of the reference study of $18 \mathrm{~g} / \mathrm{m}^{2}$ is compared to the consumption after 6 weeks of $6 \mathrm{~g} / \mathrm{m}^{2}$. Statistical analyses were performed on the intention-to-treat population defined as all randomised patients to whom the study medication was dispensed, with last observation carried forward employed to impute missing data including endpoints. For the primary efficacy analysis, the target parameter was the percentage of days on which topical corticosteroid medication was used, i.e. the number of days on topical corticosteroid medication divided by the number of days from week 0 to week 24; patients discontinuing due to lack of efficacy or adverse events were handled as if they had applied topical corticosteroids for the rest of the study. The two treatment groups were compared using the Wilcoxon rank sum test at the 5\% significance level.

For secondary efficacy analysis, tests were used in an exploratory manner. Treatment groups were compared using analysis of covariance, Wilcoxon rank sum test, logistic regression or Fisher's exact test. Survival analytical methods (log-rank test) investigating the time to first flare were also used. Cumulative survival curves investigating the time to first flare were constructed by the Kaplan-Meier method [15]. To investigate the effect of baseline variables with respect to time to first flare, a Cox proportional hazards model was fitted including the following factors: centre, baseline EASI score, baseline IGA, age category and treatment group. The EASI was analysed using an analysis of covariance.

For quality of life assessments, summary statistics were calculated at each time point for each treatment group. The percentage change from baseline was summarised in a similar manner.

Safety analysis was descriptive. Group comparisons for adverse events were performed using Fisher's exact test. For laboratory parameters, differences between treatment groups were analysed by the Wilcoxon rank sum test.

\section{Results}

\section{Patients}

Of the 192 patients from 16 centres who were randomised for treatment, 96 were randomised to the pimecrolimus-based treatment regimen and 96 to the control group. A flow diagram of patient accounting and treatment outcome is shown in figure 1. The demographic and clinical characteristics of the patients are summarised in table 1. There were no clinically or statistically significant demographic differences between the two groups at baseline. The mean affected body surface area was $17.0 \%$ in the pimecrolimus group and $16.9 \%$ in the control group. The majority of patients in each group had moderate AD at baeline ( 64.6 and $70.8 \%$ in the pimecrolimus and control groups, respectively).

More patients in the control group than in the pimecrolimus group discontinued prior to study completion (37.5 vs. $22.9 \%$, respectively). Discontinuation was due mainly to an unsatisfactory therapeutic effect (27.1 vs. $15.6 \%$, respectively). The time to discontinuation was significantly longer in the pimecrolimus group $(\mathrm{p}<0.05)$.

\section{Efficacy}

Treatment with pimecrolimus cream $1 \%$ was associated with a significant reduction in the percentage of days on which patients used corticosteroid medication (table 2). In the pimecrolimus group, patients used corticosteroids on an average (mean) of 14.2\% (95\% confidence interval, CI: 8.3-21.1) of 168 days (i.e. study duration) compared with $37.2 \%$ (95\% CI: 30.4-44.0) in the control 
Table 1. Baseline demographic and clinical characteristics of patients in the pimecrolimus and control treatment groups

\begin{tabular}{lcc}
\hline & $\begin{array}{l}\text { Pimecrolimus } 1 \% \\
(\mathrm{n}=96)\end{array}$ & $\begin{array}{c}\text { Control } \\
(\mathrm{n}=96)\end{array}$ \\
\hline Age, years & & \\
$\quad$ Mean $\pm \mathrm{SD}$ & $31.8 \pm 11.1$ & $32.5 \pm 10.7$ \\
$\quad$ Median & 28.5 & 29.5 \\
$\quad$ Range & $18-68$ & $18-69$ \\
Sex & & \\
Male & $36(37.5)$ & $41(42.7)$ \\
$\quad$ Female & $60(62.5)$ & $55(57.3)$ \\
Total body surface area involved, \% & \\
$\quad$ Mean & $17.0 \pm 7.6$ & $16.9 \pm 10.7$ \\
Range & $5.0-45.0$ & $5.0-76.0$ \\
EASI & & \\
$\quad$ Mean & $11.2 \pm 5.1$ & $10.8 \pm 6.1$ \\
$\quad$ Range & $2.0-26.6$ & $2.8-35.3$ \\
IGA score & & \\
$\quad 3$ (moderate) & $62(64.6)$ & $68(70.8)$ \\
4 (severe) & $33(34.4)$ & $28(29.2)$ \\
$\quad$ (very severe) & $1(1.0)$ & $0(0.0)$ \\
\hline
\end{tabular}

Figures in parentheses indicate percentages.

Fig. 1. Flow of participants through the trial. ITT $=$ Intention to treat.

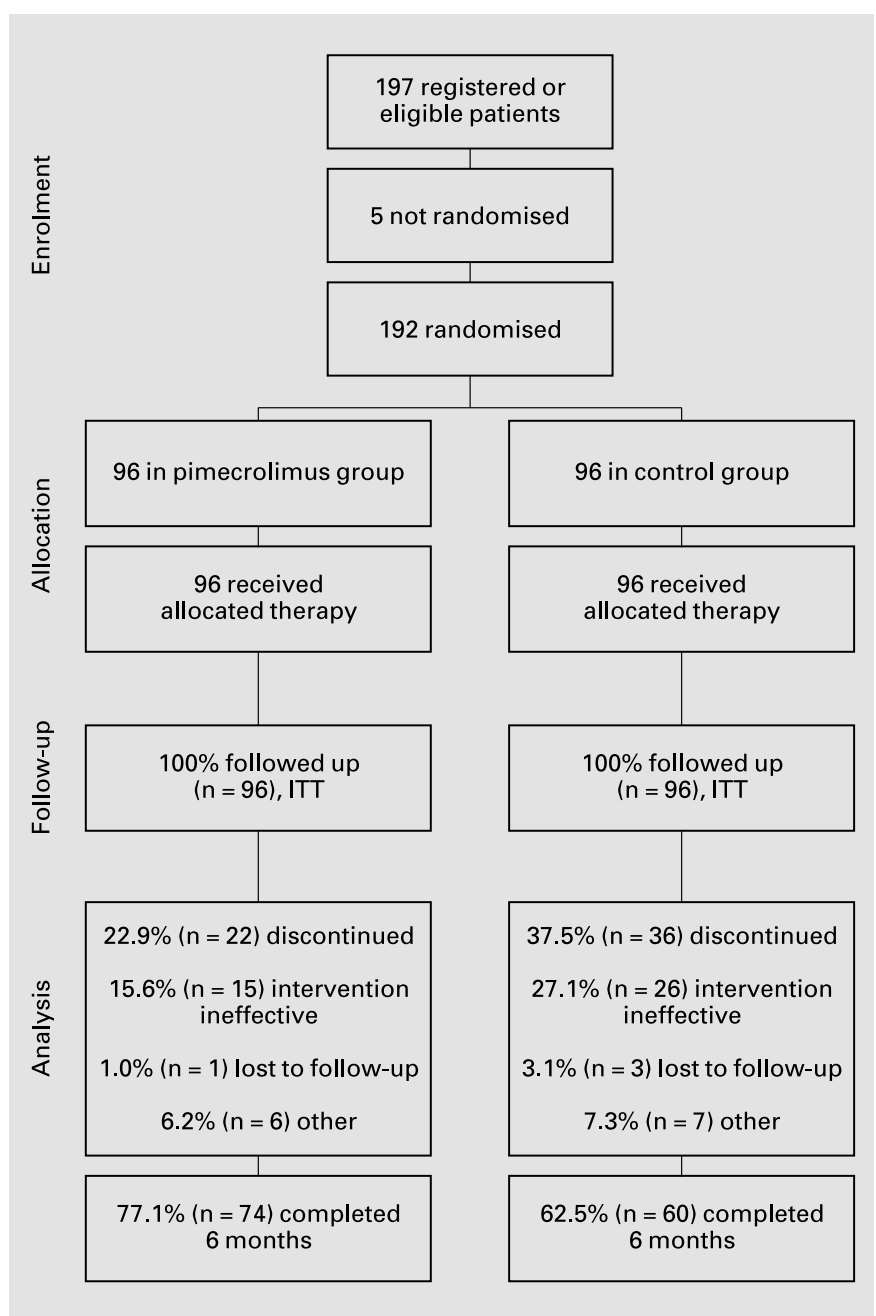

Table 2. Percentage of days of topical corticosteroid treatment by baseline disease severity

\begin{tabular}{lcrrrr}
\hline Treatment groups & $\mathrm{n}$ & Mean \pm SD & Median & Range & p value $^{1}$ \\
\hline All patients & & & & & \\
$\quad$ Pimecrolimus 1\% & 96 & $14.2 \pm 24.2$ & 2.1 & $0-97.0$ & $<0.001$ \\
$\quad$ Control & 96 & $37.2 \pm 34.6$ & 27.8 & $0-98.2$ & \\
$\begin{array}{l}\text { Moderate disease (IGA =3) } \\
\quad \text { Pimecrolimus 1\% }\end{array}$ & 62 & $9.5 \pm 19.8$ & 0.0 & $0-97.0$ & $<0.001$ \\
$\quad$ Control & 68 & $37.0 \pm 36.3$ & 23.5 & $0-98.2$ & \\
$\quad \begin{array}{l}\text { Severe disease (IGA = 4) } \\
\quad \text { Pimecrolimus 1\% }\end{array}$ & 33 & $23.1 \pm 29.5$ & 7.7 & $0-87.5$ & \\
$\quad$ Control & 28 & $37.8 \pm 30.4$ & 35.2 & $0-91.7$ & 0.027 \\
\hline
\end{tabular}

One patient with very severe disease $(\mathrm{IGA}=5)$ is not shown in the table.

1 Wilcoxon rank sum test. 


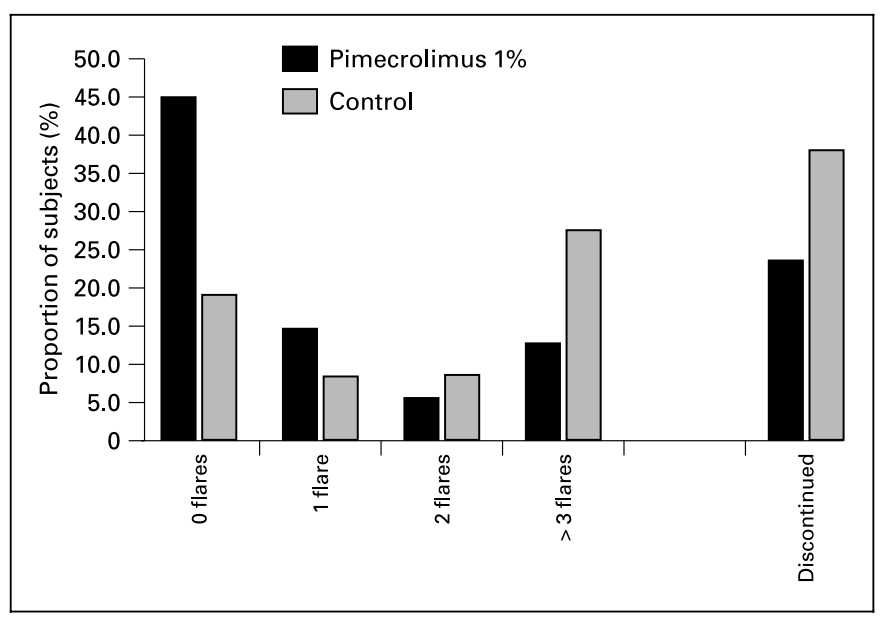

Fig. 2. Incidence of flares of $\mathrm{AD}$ in the pimecrolimus and control treatment groups during the 6-month study.

group ( $<<0.001)$. Forty-seven $(49 \%)$ of the pimecrolimus-treated patients compared to 21/96 (21.9\%) of those in the control group did not use steroids at all.

The two treatment groups also showed significant differences in the incidence of flares and the time to first flare. In the pimecrolimus group, patients experienced a mean of 1.1 (95\% CI: 0.7-1.4) flares during the 24 weeks of the study, compared to a mean of 2.4 (95\% CI: $2.0-2.8$ ) flares in the control group ( $\mathrm{p}<0.001)$. In total, $43 / 96$ $(44.8 \%)$ of patients in the pimecrolimus group had no flares in the course of the study, compared with $18 / 96$ $(18.8 \%)$ in the control group. The incidence of flares is summarised in figure 2 . The estimated median time to first flare was 144 days (95\% CI: 105-> 168) in the pimecrolimus group and 26 days (95\% CI: $21-47$ ) in the control group (fig. 3).

Based on the IGA, $79 / 96(82.3 \%)$ of patients in the pimecrolimus group showed disease improvement from baseline of at least 1 IGA score, and 66/96 (68.6\%) were classified as treatment success (IGA $\leq 2$ ). In contrast, an improvement from baseline was recorded in 49/96 $(51.0 \%)$ of the patients receiving a conventional therapy, and the success rate was $36.5 \%$ (35/96). Similar results were achieved according to the patients' self-assessment at week 24 , with $62 / 96(64.6 \%)$ of the patients in the pimecrolimus group assessing their disease as completely or well controlled compared with 34/96 (35.4\%) of the patients in the control group. Exploratory analysis of treatment differences showed statistical significance for both parameters $(\mathrm{p}<0.001)$.

Pimecrolimus Cream in the Long-Term

Management of $\mathrm{AD}$ in Adults

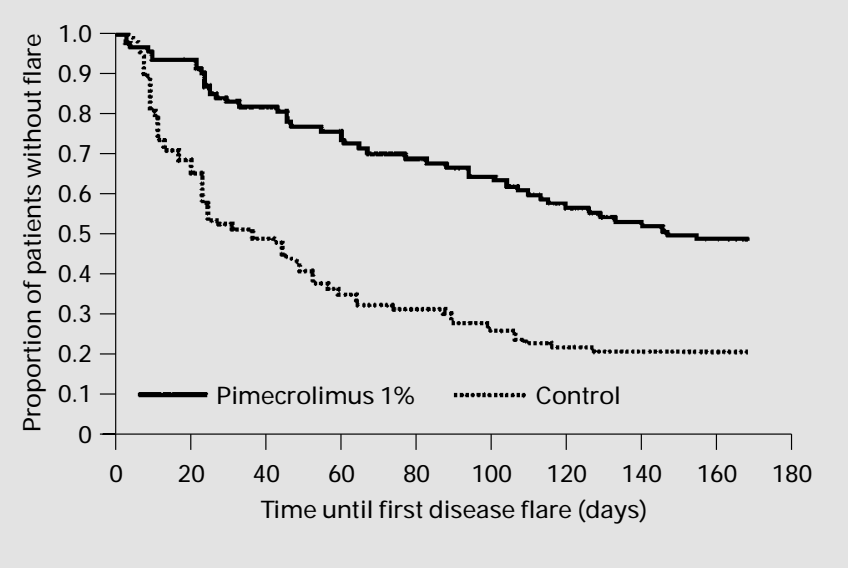

Fig. 3. Kaplan-Meier estimate of time to first flare in the pimecrolimus and control treatment groups during the 6-month study.

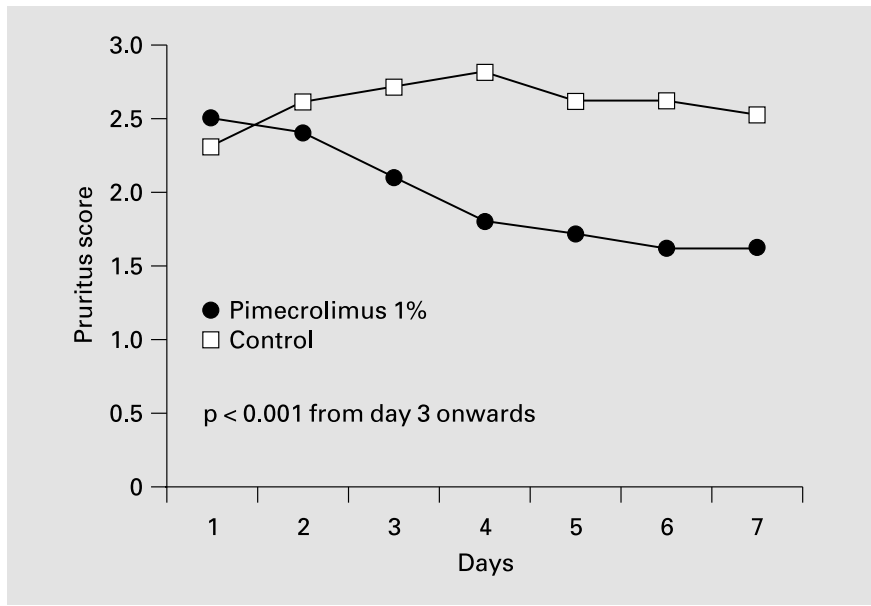

Fig. 4. Time course showing changes in pruritus scores during the first week of treatment of $\mathrm{AD}$ with pimecrolimus cream $1 \%$ and a corresponding vehicle cream. $\mathrm{p}<0.001$ from day 3 onwards.

Pruritus decreased within the first 2 days of treatment with pimecrolimus but increased in the control group (fig. 4). With the pimecrolimus-based treatment, the EASI was reduced on average by $48.3 \%$, from 11.2 to 5.7 (95\% CI: 4.1-6.9), whereas the decrease in the control group was only $15.9 \%$, from 10.8 to 8.8 (95\% CI: $7.5-10.5$; $\mathrm{p}<$ 0.001). Similar decreases were observed in the extent of body surface area affected, namely a reduction of $48.4 \%$ in the pimecrolimus group and $20.5 \%$ in the control group $(\mathrm{p}<0.01)$.

Dermatology 2002;205:271-277 


\section{Quality of Life}

Quality of life improved in both treatment groups during the 6 months of the study, but the improvement was significantly greater in the pimecrolimus group. The mean decrease (i.e. improvement) in the QoLIAD score was $25.6 \%$ in the pimecrolimus group, compared to $7.4 \%$ in the control group $(p=0.002)$. Similarly, the mean decrease (i.e. improvement) in the DLQI score in the pimecrolimus group was $22.0 \%$, compared to $6.7 \%$ in the control group $(\mathrm{p}=0.01)$.

\section{Safety}

Pimecrolimus was well tolerated, with a similar incidence of drug-related adverse events in the pimecrolimus $(24.0 \%)$ and the control group (20.8\%). Only 5 patients discontinued due to adverse events. One patient in the pimecrolimus group had an aneurysm, which was not suspected of being related to the study medication. Four patients in the control group discontinued due to adverse events possibly related to the study medication. In 3 patients, contact dermatitis was suspected and 1 patient had application site pain.

Local tolerability was good in both treatment groups. The incidence of local adverse events was $39.6 \%(38 / 96)$ in the pimecrolimus group and $36.5 \%(35 / 96)$ in the control group. More patients in the pimecrolimus group than in the control group experienced application site burning ( $10 \mathrm{vs.} 3$ patients, respectively), which resolved within 1-7 days.

In total, $18(18.8 \%)$ of pimecrolimus-treated patients and $9(9.4 \%)$ of patients in the control group had at least 1 skin infection by month 6 ; this difference was not statistically significant $(95 \% \mathrm{CI}$ for the treatment difference: -19.1 to 0.4 ). The imbalance was mainly due to a higher number of herpes infections in the pimecrolimus group (10 vs. 5 ) whereas the incidence rates of bacterial (4 vs. 3 ) and fungal ( 2 vs. 1) infections were similar. Six of 10 cases in the pimecrolimus group were herpes labialis (areas not treated with the study medication) compared with 1 of 5 in the control group; there were 2 cases of eczema herpeticum in the study, both in the control group.

No clinically relevant laboratory abnormalities were observed during the study.

\section{Discussion}

The results of this 6-month study demonstrate for the first time that pimecrolimus cream $1 \%$, used at the first signs or symptoms of $\mathrm{AD}$, reduces or eliminates the need for topical corticosteroids in adult patients.
Almost 50\% of patients in the pimecrolimus cream 1\% group did not need any corticosteroid throughout the study. When patients were stratified according to the severity of disease at baseline, a marked reduction of days requiring corticosteroid treatment was especially evident in patients with moderate disease. The effect was much more pronounced in patients with severe disease when the median value rather than the mean value was considered, since the latter is very susceptible to distortion by outliers.

The results of this study are consistent with the data of two other trials $[16,17]$, which investigated the new pimecrolimus-based treatment in children and infants. In both those studies, the primary outcome measure was the reduction in the incidence of flares, and the results were similar to the present study. In those studies, the proportion of patients experiencing no flares in 6 months in the pimecrolimus group was 67.6\% in infants (aged 3-23 months) and $61.0 \%$ in children (aged 2-17 years), compared to $45 \%$ of the adult patients in the present study. In the control groups, the respective flare-free incidences were $30.4 \%$ for infants, $34.2 \%$ for children and $19 \%$ in the present study. In all three studies the difference between the pimecrolimus-based treatment and the conventional therapy was statistically highly significant. The benefit of treatment in adults was very similar to that in children in spite of the higher incidence of flares in both groups in the present study. Infants, 3-23 months of age, seem to derive even more benefit than older children and adults from the pimecrolimus-based treatment.

The time to first flare was more than 5 times longer in the pimecrolimus group compared with the control group. This illustrates the extent of the treatment effect and has positive consequences for the patient's well-being.

There were 10 cases of application site burning in the pimecrolimus group compared with 3 in the control group. However, the events were transient and did not lead to discontinuation. Although there was no increase in the incidence of bacterial or fungal infections, herpes simplex infections were numerically more frequent in the pimecrolimus group than in the control group. However, this imbalance was not statistically significant and did not take into account the longer average time spent in the study by the patients in the pimecrolimus group. Furthermore, the imbalance was largely due to the higher number of herpes labialis infections in the pimecrolimus group; herpes labialis infections appear in areas not generally affected by $\mathrm{AD}$ and hence were not treated with the study medication (i.e. perioral). 
In conclusion, this study clearly demonstrated the benefits of the new pimecrolimus-based treatment strategy in adult $\mathrm{AD}$. When used at the first signs or symptoms of $\mathrm{AD}$, pimecrolimus prevents the condition from progressing to a flare and reduces or eliminates altogether the need for topical corticosteroids. Reserving steroids for rescue therapy will reduce the need for their long-term use and minimise the risk of steroid-associated side-effects. In addition to the steroid-sparing effect, pimecrolimus reduces the number of flares and improves overall disease control of adult AD.

\section{Acknowledgements}

The participating investigators of the CASM-DE-01 study group were M. Meurer and G. Wozel (Dresden), P. Altmeyer, C. Szliska and M. Freitag (Bochum), R. Ruzicka and L. Malek (Düsseldorf), J. Jünger, E. Piche and C. Schulz (Tübingen), W. Sterry, T. Zuberbier,
M. Worm and M. Esen (Berlin), G. Plewig, A. Wollenberg and C. Baldauf (München), M. Fartasch and M. Radulescu (Erlangen), H. Mensing and O. Hoch (Hamburg), T. Bieber, P. Hanfland, K. Steen and J. Haberstock (Bonn), P. Elsner, U. Barta (Jena), U. Peter, M. Weidmann, R. Schiener and H. Pillekamp (Ulm), M. Landthaler and T. Vogt (Regensburg), R. Fölster-Holst, I. Lange and I. Kreiselmaier (Kiel), G. Albrecht, A. Härtel and F. Garbe (Berlin), S. Müller (Münster) and K.G. Meyer (Berlin).

The authors thank G. Petry (Seligenstadt), H. Heinrich (Berlin), K. Krüger-Barvels (Niederkassel), D. Müller (Erfurt), W. Mühlbauer (Berlin), M. Ulrich, S. Seyfried and S. Nowak (Nürnberg) who were responsible for monitoring and logistics.

Contributors: M.M., G.W., G.W. and M.B. elaborated the design of the protocol. R.F.H. and M.J. investigated a high proportion of the study population and gave important feedback, which helped to optimise the conduct of the study. All authors were involved in the interpretation of the data. M.M. and M.B. wrote the draft paper, to which all authors contributed. M.M. and M.B. are the guarantors.

The study was supported by a grant from Novartis Pharma $\mathrm{GmbH}$, Nürnberg. G. Weidinger and M. Bräutigam are employees of Novartis.

\section{References}

1 Hepburn D, Yohn JJ, Weston WL: Topical steroid treatment in infants, children, and adolescents. Adv Dermatol 1994;9:225-254.

2 Raimer SS: Managing pediatric atopic dermatitis. Clin Pediatr 2000;39:1-14.

3 Charman CR, Morris AD, Williams HC: Topical corticosteroid phobia in patients with atopic eczema. Br J Dermatol 2000;142:931-936.

4 Fukaya M: Why do patients with atopic dermatitis refuse to apply topical corticosteroids? Dermatology 2000;201:242-245.

5 Hill CJ, Rosenberg A Jr: Adverse effects from topical steroids. Cutis 1978;21:624-628.

6 McLean CJ, Lobo RF, Brazier DJ: Cataracts, glaucoma, femoral avascular necrosis caused by topical corticosteroid ointment. Lancet 1995;345:330

7 Meingassner JG, Grassberger M, Fahrngruber H, Moore HD, Schuurman H, Stütz A: A novel anti-inflammatory drug, SDZ ASM 981, for the topical and oral treatment of skin diseases: In vivo pharmacology. Br J Dermatol 1997;137: 568-576.

8 Hultsch T, Muller KD, Meingassner JG, Grassberger M, Schopf RE, Knop J: Ascomycin macrolactam derivative SDZ ASM 981 inhibits the release of granule-associated mediators and of newly synthesized cytokines in RBL $2 \mathrm{H} 3$ mast cells in an immunophilin-dependent manner. Arch Dermatol Res 1998;290:501507.
9 Grassberger M, Baumruker T, Enz A, Hiestand P, Hultsch T, Kalthoff F, Schuler W, Schultz M, Werner FJ, Winiski A, Wolff B, Zenke G: A novel anti-inflammatory drug, SDZ ASM 981, for the treatment of skin diseases: In vitro pharmacology. Br J Dermatol 1999;141:264-273.

10 Queille-Roussel C, Paul C, Duteil L, Lefebvre MC, Rapatz G, Zagula M, Ortonne JP: The new topical ascomycin derivative SDZ ASM 981 does not induce skin atrophy when applied to normal skin for 4 weeks: A randomized, double-blind controlled study. Br J Dermatol 2001; 144:507-513.

11 Rajka G: Natural history and clinical manifestations of atopic eczema. Clin Rev Allergy 1986;4:3-26.

12 Hanifin JM, Thurston M, Omoto M, Cherill R, Tofte SJ, Graeber M: The eczema area and severity index (EASI): Assessment of reliability in atopic dermatitis. EASI Evaluator Group. Exp Dermatol 2001;10:11-18.

13 Finlay AY, Khan GK: Dermatology Life Quality Index (DLQI) - A simple practical measure for routine clinical use. Clin Exp Dermatol 1994;19:210-216.
14 Boehmer S, Naujoks C, Kohlmann T: Gesundheitsbezogene Lebensqualität - Entwicklung eines Fragebogens für Neurodermitis-Patienten. Hautfreund 2001;2:30-33.

15 Kaplan EL, Meier P: Nonparametric estimation from incomplete samples. J Am Stat Assoc 1958;53:457-481.

16 Kapp A, Papp K, Bingham A, Fölster-Holst R, Ortonne J-P, Potter PC, Gulliver W, Paul C, Molloy S, Barbier N, Thurston M, de Prost Y, for the FREE (Infants) Multicenter Investigator Study Group: Long-term management of atopic dermatitis in infants with topical pimecrolimus, a non-steroid anti-inflammatory drug. J Allergy Clin Immunol 2002;110:277284.

17 Wahn U, Bos JD, Goodfield M, Caputo R, Papp K, Manjra A, Dobozy A, Paul C, Molloy S, Hultsch T, Graeber M, Cherill R, de Prost Y, for the FREE (Children) Multicenter Investigator Study Group: Efficacy and safety of pimecrolimus cream in the long-term management of atopic dermatitis in children. Pediatrics 2002;110:e2. 